\title{
A Chromogenic Macrocycle Exhibiting Cation-Selective and Anion-Controlled Color Change: An Approach to Understanding Structure-Color Relationships
}

\author{
Soo Jin Lee, ${ }^{\dagger}$ Jong Hwa Jung, ${ }^{*, \dagger}$ Joobeom Seo, ${ }^{\dagger}$ II Yoon, ${ }^{\dagger}$ Ki-Min Park, ${ }^{\dagger}$ Leonard F. Lindoy, ${ }^{\ddagger}$ \\ and Shim Sung Lee ${ }^{*, \dagger}$ \\ 'Department of Chemistry and Research Institute of Natural Sciences, Gyeongsang National \\ University, Chinju660-701, Korea.E-mail:sslee@gsnu.ac.kr \\ ${ }^{\ddagger}$ Centre for Heavy Metal Research, School of Chemistry, F11, The University of Sydney, NSW 2006, \\ Australia.
}

\section{Table of Contents:}

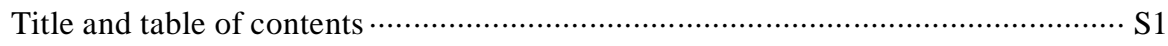

Scheme S1. Synthesis of the azo-coupled macrocycle 1 ….................................. S2

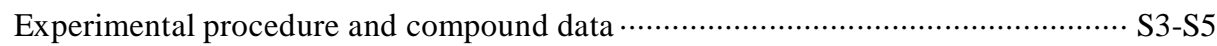

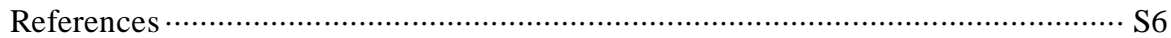

Table S1. Crystal data and structure refinement for 2, $\left[\mathrm{Hg}(\mathbf{1}) \mathrm{ClO}_{4}\right]\left(\mathrm{ClO}_{4}\right) \cdots \cdots \cdots \cdots \cdots . . . \mathrm{S} 7$

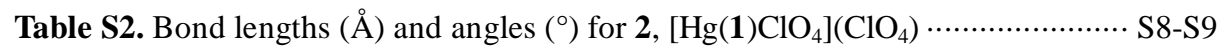

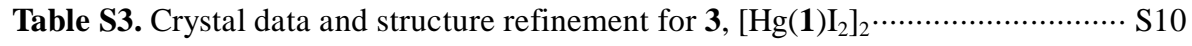

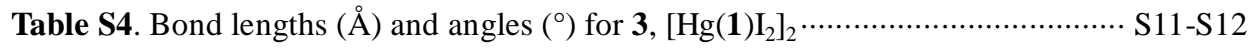

Figure S1. Metal-induced color changes of 1 in acetonitrile ….......................... S13

Figure S2. UV/Vis titrations of $1(0.30 \mathrm{mM})$ with

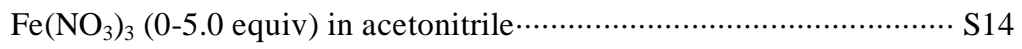

Figure S3. UV/Vis titrations of $1(0.30 \mathrm{mM})$ with

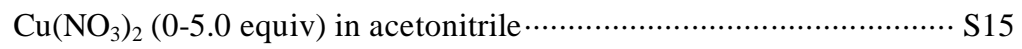

Figure S4. Anion-controlled color changes of $\mathbf{1}$ in acetonitrile …..................... S16

Figure S5. UV/Vis titrations of $1(0.30 \mathrm{mM})$ with

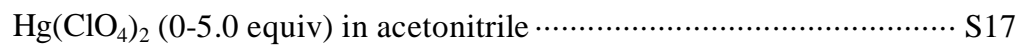

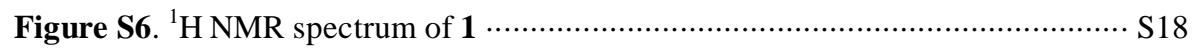

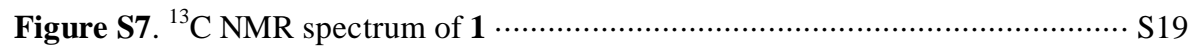

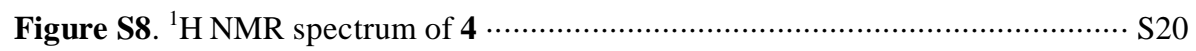

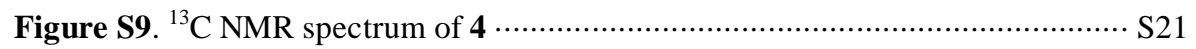




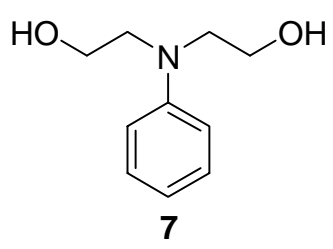

a)

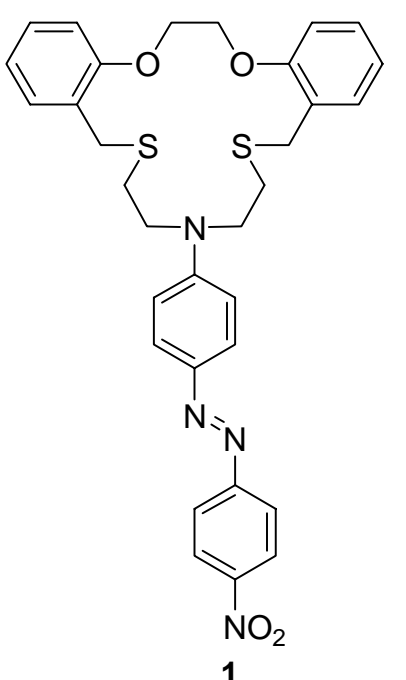

b)<smiles>SCCN(CCS)c1ccccc1</smiles>

5

c)

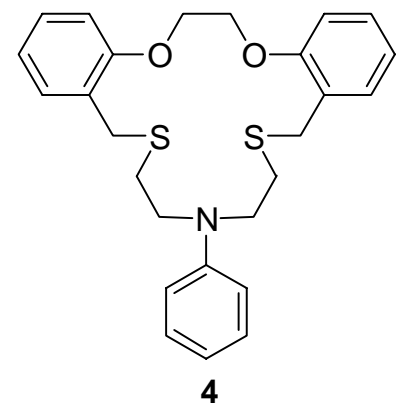

Scheme S1. Synthesis of the azo-coupled macrocycle 1: a) $p$-TsCl, pyridine, $82 \%$; b) thiourea, $\mathrm{NaHCO}_{3}, \mathrm{EtOH}, \mathrm{H}_{2} \mathrm{O}$; c) 1,2-bis(2-(chloromethyl)phenoxy)ethane, $\mathrm{Cs}_{2} \mathrm{CO}_{3}$, DMF, 48\%; d) diazonium salt, DMF, $88 \%$. 
To a solution of $N, N^{\prime}$-phenyldiethanolamine $7(25.0 \mathrm{~g}, 138 \mathrm{mmol})$ in dry pyridine $(33 \mathrm{~mL})$ at $0{ }^{\circ} \mathrm{C}$ was slowly added para-toluenesulfonyl chloride $(31.5 \mathrm{~mL}, 333 \mathrm{mmol})$. As the reaction mixture was allowed to reach $\mathrm{rt}$, the reaction became slightly exothermic. After $3 \mathrm{~h}$, the clear yellow mixture was slowly poured into $500 \mathrm{~mL}$ of fast stirring ice-water, giving a solid. Recrystallization from ethanol/toluene yielded 6, 2,2'-(phenylazanediyl)bis(ethane-2,1-diyl)bis(4methylbenzenesulfonate) as a colorless crystalline solid (55.21 g, 81.7\%). The analysis data of $\mathbf{6}$ obtained from different route have been reported [J. Organometal. Chem., 2001, 626, 221-226].

To a solution of $6(30.0 \mathrm{~g}, 67.2 \mathrm{mmol})$ in ethanol $(100 \mathrm{~mL})$, thiourea $(9.32 \mathrm{~g}, 122.54 \mathrm{mmol})$ was added. The reaction mixture was refluxed for $2 \mathrm{~h}$, then concentrated and saturated $\mathrm{NaHCO}_{3}(a q .60 \mathrm{~mL})$ was added. The mixture was refluxed for $3 \mathrm{~h}$. After cooling to $\mathrm{rt}, \mathrm{CHCl}_{3}(50 \mathrm{~mL})$ were added, and the organic layer was separated and dried over $\mathrm{MgSO}_{4}$. Removal of the organic solvent in vacuo afforded 5, 2,2'-(phenylazanediyl) diethanethiol as a colorless oil $(87 \%)$. Due to the instability of $\mathbf{5}$, no further purification was done.

4 was synthesized under high dilution employing the cyclization procedure. Cesium carbonate $(5.70 \mathrm{~g}, 17.50 \mathrm{mmol})$ was dissolved in DMF (1500 mL) in a 3-L round-bottom flask. 5 (4.0 g, $13.46 \mathrm{mmol})$ and 1,2-bis(2-(chloromethyl) phenoxy) ethane (10.05 g, $47.1 \mathrm{mmol})$ were dissolved in DMF $(30 \mathrm{~mL})$ and placed in a 50-mL glass syringe. Under a nitrogen atmosphere, the contents of the syringe were added dropwise at regular speed (a rate of $10 \mathrm{~mL} \mathrm{~h}$ ) into the DMF solution by the aid of microprocessor controlled syringe pump at $45-50{ }^{\circ} \mathrm{C}$ for $5 \mathrm{~h}$. The mixture was kept for a further $10 \mathrm{~h}$. After cooling to rt the reaction mixture was filtered and evaporated. Water (100 $\mathrm{mL})$ was added, and the mixture was extracted with $3 \times 70 \mathrm{~mL}$ of dichloromethane. The organic phase was dried over anhydrous sodium sulfate, filtered and the solvent was removed to give a yellow oil. Flash column chromatography $\left(\mathrm{SiO}_{2}, n\right.$-hexane : dichloromethane $\left./ 1: 1, \mathrm{R}_{f}=0.3\right)$ afforded the product as a white solid in $48 \%$ yield $(2.75 \mathrm{~g})$. m.p. $124{ }^{\circ} \mathrm{C} ;{ }^{1} \mathrm{H} \mathrm{NMR}(500$ $\left.\mathrm{MHz}, \mathrm{CDCl}_{3}, 25{ }^{\circ} \mathrm{C}, \mathrm{TMS}\right) \delta=7.43\left(\mathrm{~d},{ }^{2} J(\mathrm{H}, \mathrm{H})=7.5 \mathrm{~Hz}, 2 \mathrm{H} ; \mathrm{Ar}-\mathrm{H}\right), 7.23\left(\mathrm{t},{ }^{3} J(\mathrm{H}, \mathrm{H})=5.5 \mathrm{~Hz}, 2 \mathrm{H} ; \mathrm{Ar}-\mathrm{H}\right), 7.12\left(\mathrm{t},{ }^{3} J\right.$ $(\mathrm{H}, \mathrm{H})=7.5 \mathrm{~Hz}, 2 \mathrm{H} ; \mathrm{Ar}-\mathrm{H}), 6.98\left(\mathrm{~d},{ }^{2} J(\mathrm{H}, \mathrm{H})=5.5 \mathrm{~Hz}, 2 \mathrm{H} ; \mathrm{Ar}-\mathrm{H}\right), 6.93\left(\mathrm{t},{ }^{3} J(\mathrm{H}, \mathrm{H})=7.0 \mathrm{~Hz}, 2 \mathrm{H} ; \mathrm{Ar}-\mathrm{H}\right), 6.62\left(\mathrm{t},{ }^{3} J\right.$ $(\mathrm{H}, \mathrm{H})=7.0 \mathrm{~Hz}, 1 \mathrm{H} ; \mathrm{Ar}-\mathrm{H}), 6.51\left(\mathrm{~d},{ }^{2} J(\mathrm{H}, \mathrm{H})=8.0 \mathrm{~Hz}, 2 \mathrm{H} ; \mathrm{Ar}-\mathrm{H}\right), 4.39\left(\mathrm{~s}, 4 \mathrm{H} ; \mathrm{CH}_{2}\right), 3.87\left(\mathrm{~s}, 4 \mathrm{H} ; \mathrm{CH}_{2}\right), 3.52\left(\mathrm{t},{ }^{3} J(\mathrm{H}, \mathrm{H})\right.$ $\left.=7.5 \mathrm{~Hz}, 4 \mathrm{H} ; \mathrm{CH}_{2}\right), 2.61\left(\mathrm{t},{ }^{3} J(\mathrm{H}, \mathrm{H})=7.5 \mathrm{~Hz}, 4 \mathrm{H} ; \mathrm{CH}_{2}\right) ;{ }^{13} \mathrm{C} \mathrm{NMR}\left(125 \mathrm{MHz}, \mathrm{CDCl}_{3}, 25{ }^{\circ} \mathrm{C}, \mathrm{TMS}\right) \delta=156.4,146.7$, 130.9, 129.3, 128.3, 127.5, 121.6, 116.1, 111.9, 67.6, 51.9, 29.1, 28.6; IR (KBr): $v=1343 \mathrm{~cm}^{-1}(\mathrm{Ar}-\mathrm{N}), 1256 \mathrm{~cm}{ }^{-1}(\mathrm{C}-\mathrm{N})$; 
HRMS: m/z (\%): 451.1639 [M $\mathrm{M}^{+}$: Cald for $\mathrm{C}_{26} \mathrm{H}_{29} \mathrm{NO}_{2} \mathrm{~S}_{2} 451.1640$; Elemental analysis calcd (\%) for $\mathrm{C}_{26} \mathrm{H}_{29} \mathrm{NO}_{2} \mathrm{~S}_{2}$ : C 69.14, H 6.47, N 3.10, S 14.20; found: C 68.92, H 5.98, N 3.03, S 13.94.

1 was synthesized from reaction of 4 and diazonium salt. The diazonium salt of 4 -nitroaniline $(0.2 \mathrm{~g}, 1.44 \mathrm{mmol})$ was prepared by adding an aqueous solution of sodium nitrite $(0.13 \mathrm{~g}, 1.87 \mathrm{mmol})$ dropwise into a homogeneous mixture of $0.03 \mathrm{~mL}$ of sulfuric acid and $1 \mathrm{~mL}$ of glacial acetic acid. The mixture was stirred at $0{ }^{\circ} \mathrm{C}$ for $5 \mathrm{~min}$. The diazonium salt solution was added dropwise into a solution of $4(0.50 \mathrm{~g}, 1.11 \mathrm{mmol})$ in $50 \mathrm{~mL}$ of DMF at $0{ }^{\circ} \mathrm{C}$. The solution was stirred for an additional $12 \mathrm{~h}$ at $0{ }^{\circ} \mathrm{C} . \mathrm{CHCl}_{3}(50 \mathrm{~mL})$ and water $(50 \mathrm{~mL})$ were added, and the organic layer was separated and dried over $\mathrm{MgSO}_{4}$. Removal of the organic solvent in vacuo afforded a red oil. Column chromatography on silica gel with $n$-hexane: dichloromethane $(1: 3)$ as eluent $\left(R_{f}=0.18\right)$ provided $\mathbf{1}$ as a red crystalline solid in $88 \%$ yield. m.p. $150{ }^{\circ} \mathrm{C} ;{ }^{1} \mathrm{H}$ NMR $\left(500 \mathrm{MHz}, \mathrm{CDCl}_{3}, 25{ }^{\circ} \mathrm{C}, \mathrm{TMS}\right) \delta=8.34\left(\mathrm{~d},{ }^{2} J(\mathrm{H}, \mathrm{H})=9.0 \mathrm{~Hz}, 2 \mathrm{H} ; \mathrm{Ar}-\mathrm{H}\right), 7.93\left(\mathrm{~d},{ }^{2} J(\mathrm{H}, \mathrm{H})=9.0 \mathrm{~Hz}\right.$, $2 \mathrm{H} ; \mathrm{Ar}-\mathrm{H}), 7.83\left(\mathrm{~d},{ }^{2} J(\mathrm{H}, \mathrm{H})=9.0 \mathrm{~Hz}, 2 \mathrm{H} ; \mathrm{Ar}-\mathrm{H}\right), 7.48\left(\mathrm{~d},{ }^{2} J(\mathrm{H}, \mathrm{H})=7.5 \mathrm{~Hz}, 2 \mathrm{H} ; \mathrm{Ar}-\mathrm{H}\right), 7.28(\mathrm{t}, 2 \mathrm{H} ; \mathrm{Ar}-\mathrm{H}), 7.05\left(\mathrm{t},{ }^{3} J\right.$ $(\mathrm{H}, \mathrm{H})=7.5 \mathrm{~Hz}, 2 \mathrm{H} ; \mathrm{Ar}-\mathrm{H}), 7.03\left(\mathrm{t},{ }^{3} J(\mathrm{H}, \mathrm{H})=7.0 \mathrm{~Hz}, 2 \mathrm{H} ; \mathrm{Ar}-\mathrm{H}\right), 6.60\left(\mathrm{~d},{ }^{2} J(\mathrm{H}, \mathrm{H})=9.5 \mathrm{~Hz}, 2 \mathrm{H} ; \mathrm{Ar}-\mathrm{H}\right), 4.40(\mathrm{~s}, 4 \mathrm{H}$; $\left.\mathrm{CH}_{2}\right), 3.91\left(\mathrm{~s}, 4 \mathrm{H} ; \mathrm{CH}_{2}\right), 3.71\left(\mathrm{t},{ }^{3} J(\mathrm{H}, \mathrm{H})=7.5 \mathrm{~Hz}, 4 \mathrm{H} ; \mathrm{CH}_{2}\right), 2.61\left(\mathrm{t},{ }^{3} J(\mathrm{H}, \mathrm{H})=7.5 \mathrm{~Hz}, 4 \mathrm{H} ; \mathrm{CH}_{2}\right) ;{ }^{13} \mathrm{C} \mathrm{NMR}(125 \mathrm{MHz}$, $\left.\mathrm{CDCl}_{3}, 25{ }^{\circ} \mathrm{C}, \mathrm{TMS}\right) \delta=156.8,156.4,150.5,143.9,131.0,128.5,127.0,126.2,124.7,122.6,121.8,112.0,111.6,67.7$ 52.2, 29.0, 28.2; IR (KBr): $v=1517,1328 \mathrm{~cm}^{-1}\left(\mathrm{NO}_{2}\right), 1387 \mathrm{~cm}^{-1}(\mathrm{Ar}-\mathrm{N}), 1241 \mathrm{~cm}^{-1}(\mathrm{C}-\mathrm{N}) ; \mathrm{HRMS}: \mathrm{m} / \mathrm{z}(\%): 600.1868$ $\left[\mathrm{M}^{+}\right]$: Cald for $\mathrm{C}_{32} \mathrm{H}_{32} \mathrm{~N}_{4} \mathrm{O}_{4} \mathrm{~S}_{2}$ 600.1865; Elemental analysis calcd (\%) for $\mathrm{C}_{32} \mathrm{H}_{32} \mathrm{~N}_{4} \mathrm{O}_{4} \mathrm{~S}_{2}$ : C 63.98, H 5.37, N 9.33, S 10.67; found: C 64.01, H 5.06, N 9.04, S 10.62.

Pale-yellow complex 2, $\left[\mathrm{Hg}(\mathbf{1}) \mathrm{ClO}_{4}\right]\left(\mathrm{ClO}_{4}\right)$ was prepared as following. Benzene $(1 \mathrm{~mL})$ was layered above dichloromathane solution $(3 \mathrm{~mL})$ of $1(25 \mathrm{mg}, 41.6 \mathrm{mmol})$ and then methanol solution $(3 \mathrm{~mL}) \mathrm{of} \mathrm{Hg}\left(\mathrm{ClO}_{4}\right)_{2}(14.3 \mathrm{mg}$, $41.6 \mathrm{mmol}$ ) was layered on the benzene. On standing for a week at $\mathrm{rt}$ the pale-yellow crystals of 2 suitable for X-ray analysis was afforded.

Red-colored complex 3, $\left[\mathrm{Hg}(\mathbf{1}) \mathrm{I}_{2}\right]_{2}$ was prepared as following. A methanol solution $(3 \mathrm{~mL})$ of $\mathrm{HgI}_{2}(18.9 \mathrm{mg}$, 41.6mmol) was layered above dichloromathane solution ( $3 \mathrm{~mL})$ of 1 ( $25 \mathrm{mg}, 41.6 \mathrm{mmol})$. On standing for a week at $\mathrm{rt}$ the red crystals of $\mathbf{3}$ suitable for X-ray analysis was afforded. 


\section{Crystallographic Structure Determinations}

All data were collected on a Bruker Smart diffractometer equipped with a graphite monochromated $\mathrm{Mo} \mathrm{K} \alpha(\lambda=$ $0.71073 \AA$ ) radiation source and a CCD detector. The 45 frames of two dimensional diffraction images were collected and processed to obtain the cell parameters and orientation matrix. The first 50 frames were retaken after complete data collection. The crystal showed no significant decay. The frame data were processed to give structure factors using the SAINT. ${ }^{1}$ The structure was solved by direct methods and refined by full matrix least squares methods on $F^{2}$ for all data using SHELXTL software. ${ }^{2}$ The non-hydrogen atoms were refined anisotropically. The carbon atom C19 in 2 show relatively large thermal displacement parameters; attempts to resolve the atoms as partial occupancy could not be done sensibly. The hydrogen atoms were placed in calculated positions and refined with a riding model with $U_{\text {iso }}$ constrained to be 1.2 times $U_{\text {eq }}$ of the parent atom. 


\section{References}

1. Bruker, SMART and SAINT: Area Detector Control and Integration Software Ver. 5.0; Bruker Analytical X-ray Instruments: Madison, Wisconsin, 1998.

2. Bruker, SHELXTL: Structure Determination Programs Ver. 5.16; Bruker Analytical X-ray Instruments: Madison, Wisconsin, 1998. 
Table S1. Crystal data and structure refinement for $\mathbf{2}$, $\left[\mathrm{Hg}(\mathbf{1}) \mathrm{ClO}_{4}\right]\left(\mathrm{ClO}_{4}\right)$

Empirical formula

Formula weight

Temperature

Wavelength

Crystal system

Space group

Unit cell dimensions

Volume

$\mathrm{Z}$

Density (calculated)

Absorption coefficient

$\mathrm{F}(000)$

Crystal size

Theta range for data collection

Index ranges

Reflections collected

Independent reflections

Completeness to theta $=27.00^{\circ}$

Absorption correction

Refinement method

Data / restraints / parameters

Goodness-of-fit on $\mathrm{F}^{2}$

Final $\mathrm{R}$ indices [I $>2 \operatorname{sigma}(\mathrm{I})]$

$\mathrm{R}$ indices (all data)

Largest diff. peak and hole
$\mathrm{C}_{32} \mathrm{H}_{32} \mathrm{Cl}_{2} \mathrm{Hg} \mathrm{N}_{4} \mathrm{O}_{12} \mathrm{~S}_{2}$

1000.23

173(2) K

$0.71073 \AA$

Monoclinic

$\mathrm{C} 2 / \mathrm{c}$

$\mathrm{a}=22.880(5) \AA \quad \alpha=90^{\circ}$.

$\mathrm{b}=25.666(6) \AA \quad \beta=123.552(5)^{\circ}$.

$\mathrm{c}=14.578(3) \AA \quad \gamma=90^{\circ}$.

7134(3) $\AA^{3}$

8

$1.862 \mathrm{Mg} / \mathrm{m}^{3}$

$4.651 \mathrm{~mm}^{-1}$

3952

$0.30 \times 0.10 \times 0.10 \mathrm{~mm}^{3}$

1.33 to $27.00^{\circ}$.

$-29<=\mathrm{h}<=27,-29<=\mathrm{k}<=32,-18<=\mathrm{l}<=18$

20435

$7418[\mathrm{R}(\mathrm{int})=0.1551]$

$95.2 \%$

Empirical SADABS

Full-matrix least-squares on $\mathrm{F}^{2}$

7418 / 0 / 478

0.992

$\mathrm{R} 1=0.0622, \mathrm{wR} 2=0.1254$

$\mathrm{R} 1=0.2073, \mathrm{wR} 2=0.1982$

1.005 and -2.485 e. $\AA^{-3}$ 
Table S2. Bond lengths $(\AA)$ and angles $\left(^{\circ}\right)$ for $\mathbf{2},\left[\mathrm{Hg}(\mathbf{1}) \mathrm{ClO}_{4}\right]\left(\mathrm{ClO}_{4}\right)$

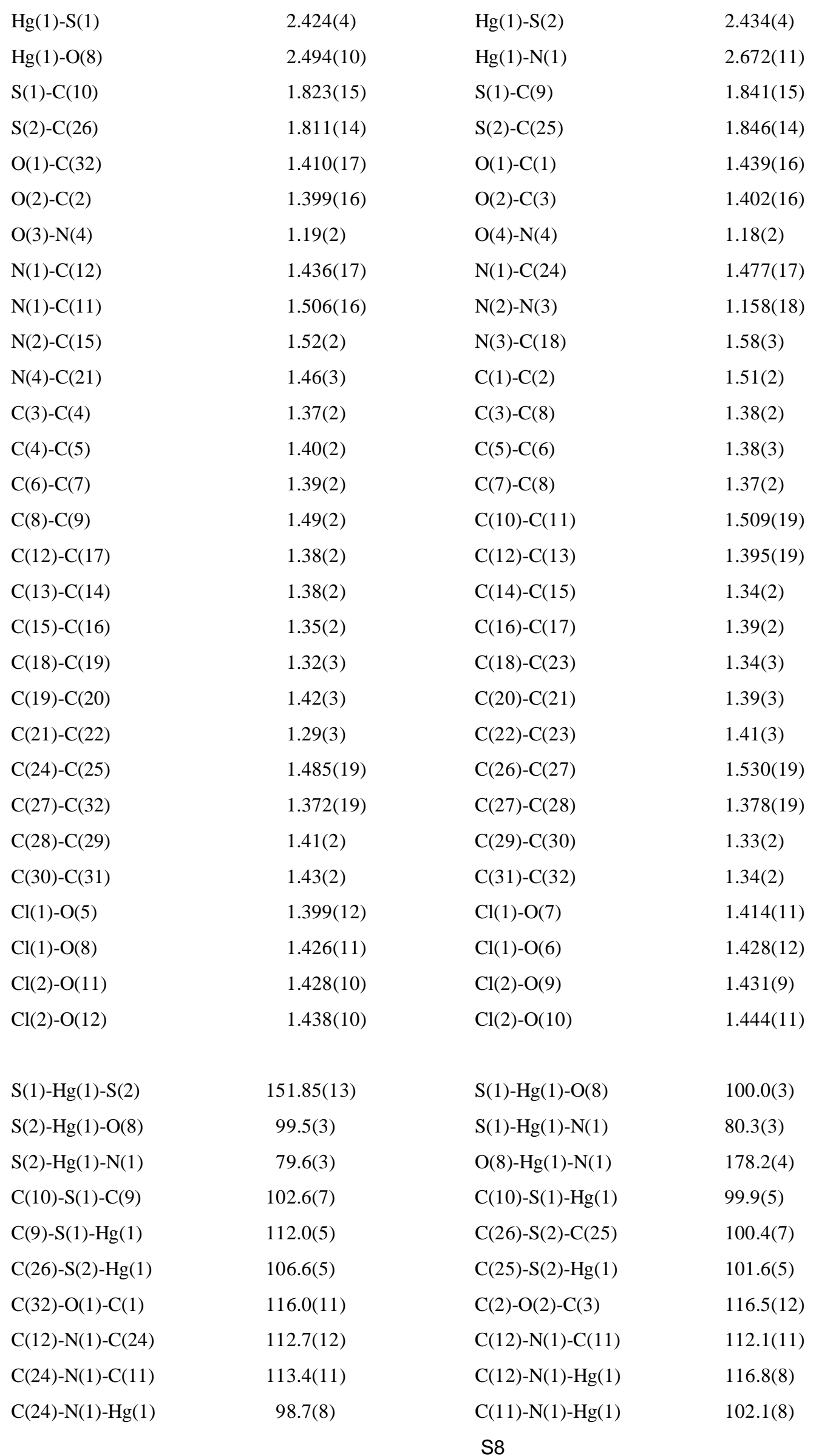




\begin{tabular}{|c|c|c|c|}
\hline $\mathrm{N}(3)-\mathrm{N}(2)-\mathrm{C}(15)$ & $101.4(18)$ & $\mathrm{N}(2)-\mathrm{N}(3)-\mathrm{C}(18)$ & $103.0(18)$ \\
\hline $\mathrm{O}(4)-\mathrm{N}(4)-\mathrm{O}(3)$ & $125(3)$ & $\mathrm{O}(4)-\mathrm{N}(4)-\mathrm{C}(21)$ & $119(3)$ \\
\hline $\mathrm{O}(3)-\mathrm{N}(4)-\mathrm{C}(21)$ & $116(3)$ & $\mathrm{O}(1)-\mathrm{C}(1)-\mathrm{C}(2)$ & $108.0(12)$ \\
\hline $\mathrm{O}(2)-\mathrm{C}(2)-\mathrm{C}(1)$ & $109.1(13)$ & $\mathrm{C}(4)-\mathrm{C}(3)-\mathrm{C}(8)$ & $122.2(15)$ \\
\hline $\mathrm{C}(4)-\mathrm{C}(3)-\mathrm{O}(2)$ & $122.6(15)$ & $\mathrm{C}(8)-\mathrm{C}(3)-\mathrm{O}(2)$ & $115.1(15)$ \\
\hline $\mathrm{C}(3)-\mathrm{C}(4)-\mathrm{C}(5)$ & $121.0(17)$ & $\mathrm{C}(6)-\mathrm{C}(5)-\mathrm{C}(4)$ & 117.1(19) \\
\hline$C(5)-C(6)-C(7)$ & $120.9(17)$ & $C(8)-C(7)-C(6)$ & $121.6(17)$ \\
\hline $\mathrm{C}(7)-\mathrm{C}(8)-\mathrm{C}(3)$ & 117.1(16) & $\mathrm{C}(7)-\mathrm{C}(8)-\mathrm{C}(9)$ & $120.9(16)$ \\
\hline $\mathrm{C}(3)-\mathrm{C}(8)-\mathrm{C}(9)$ & $121.9(14)$ & $\mathrm{C}(8)-\mathrm{C}(9)-\mathrm{S}(1)$ & $117.6(11)$ \\
\hline $\mathrm{C}(11)-\mathrm{C}(10)-\mathrm{S}(1)$ & $112.7(10)$ & $\mathrm{N}(1)-\mathrm{C}(11)-\mathrm{C}(10)$ & $111.7(11)$ \\
\hline $\mathrm{C}(17)-\mathrm{C}(12)-\mathrm{C}(13)$ & $115.9(15)$ & $\mathrm{C}(17)-\mathrm{C}(12)-\mathrm{N}(1)$ & $125.3(13)$ \\
\hline $\mathrm{C}(13)-\mathrm{C}(12)-\mathrm{N}(1)$ & $118.8(14)$ & $\mathrm{C}(14)-\mathrm{C}(13)-\mathrm{C}(12)$ & $121.2(16)$ \\
\hline $\mathrm{C}(15)-\mathrm{C}(14)-\mathrm{C}(13)$ & $119.9(17)$ & $\mathrm{C}(14)-\mathrm{C}(15)-\mathrm{C}(16)$ & $122.5(18)$ \\
\hline $\mathrm{C}(14)-\mathrm{C}(15)-\mathrm{N}(2)$ & $108.4(16)$ & $\mathrm{C}(16)-\mathrm{C}(15)-\mathrm{N}(2)$ & 129.1(19) \\
\hline$C(15)-C(16)-C(17)$ & $117.1(17)$ & $\mathrm{C}(12)-\mathrm{C}(17)-\mathrm{C}(16)$ & $123.4(15)$ \\
\hline $\mathrm{C}(19)-\mathrm{C}(18)-\mathrm{C}(23)$ & $120(2)$ & $\mathrm{C}(19)-\mathrm{C}(18)-\mathrm{N}(3)$ & $122(2)$ \\
\hline $\mathrm{C}(23)-\mathrm{C}(18)-\mathrm{N}(3)$ & $117(2)$ & $\mathrm{C}(18)-\mathrm{C}(19)-\mathrm{C}(20)$ & $122(2)$ \\
\hline $\mathrm{C}(21)-\mathrm{C}(20)-\mathrm{C}(19)$ & $118(2)$ & $\mathrm{C}(22)-\mathrm{C}(21)-\mathrm{C}(20)$ & $119(2)$ \\
\hline $\mathrm{C}(22)-\mathrm{C}(21)-\mathrm{N}(4)$ & $119(2)$ & $\mathrm{C}(20)-\mathrm{C}(21)-\mathrm{N}(4)$ & $122(3)$ \\
\hline$C(21)-C(22)-C(23)$ & $124(2)$ & $\mathrm{C}(18)-\mathrm{C}(23)-\mathrm{C}(22)$ & $118(2)$ \\
\hline $\mathrm{N}(1)-\mathrm{C}(24)-\mathrm{C}(25)$ & $112.7(11)$ & $\mathrm{C}(24)-\mathrm{C}(25)-\mathrm{S}(2)$ & $113.6(11)$ \\
\hline $\mathrm{C}(27)-\mathrm{C}(26)-\mathrm{S}(2)$ & $108.2(10)$ & $\mathrm{C}(32)-\mathrm{C}(27)-\mathrm{C}(28)$ & $117.9(14)$ \\
\hline $\mathrm{C}(32)-\mathrm{C}(27)-\mathrm{C}(26)$ & $122.0(13)$ & $\mathrm{C}(28)-\mathrm{C}(27)-\mathrm{C}(26)$ & $119.9(14)$ \\
\hline $\mathrm{C}(27)-\mathrm{C}(28)-\mathrm{C}(29)$ & 119.1(15) & $\mathrm{C}(30)-\mathrm{C}(29)-\mathrm{C}(28)$ & $122.7(16)$ \\
\hline $\mathrm{C}(29)-\mathrm{C}(30)-\mathrm{C}(31)$ & 117.1(16) & $\mathrm{C}(32)-\mathrm{C}(31)-\mathrm{C}(30)$ & $119.9(15)$ \\
\hline$C(31)-C(32)-C(27)$ & $123.0(15)$ & $\mathrm{C}(31)-\mathrm{C}(32)-\mathrm{O}(1)$ & $123.1(13)$ \\
\hline $\mathrm{C}(27)-\mathrm{C}(32)-\mathrm{O}(1)$ & $113.8(13)$ & $\mathrm{O}(5)-\mathrm{Cl}(1)-\mathrm{O}(7)$ & 107.1(9) \\
\hline $\mathrm{O}(5)-\mathrm{Cl}(1)-\mathrm{O}(8)$ & $110.0(9)$ & $\mathrm{O}(7)-\mathrm{Cl}(1)-\mathrm{O}(8)$ & $111.0(7)$ \\
\hline $\mathrm{O}(5)-\mathrm{Cl}(1)-\mathrm{O}(6)$ & $109.9(10)$ & $\mathrm{O}(7)-\mathrm{Cl}(1)-\mathrm{O}(6)$ & $110.8(8)$ \\
\hline $\mathrm{O}(8)-\mathrm{Cl}(1)-\mathrm{O}(6)$ & $108.1(8)$ & $\mathrm{Cl}(1)-\mathrm{O}(8)-\mathrm{Hg}(1)$ & $128.5(7)$ \\
\hline $\mathrm{O}(11)-\mathrm{Cl}(2)-\mathrm{O}(9)$ & $108.0(7)$ & $\mathrm{O}(11)-\mathrm{Cl}(2)-\mathrm{O}(12)$ & 109.7(7) \\
\hline $\mathrm{O}(9)-\mathrm{Cl}(2)-\mathrm{O}(12)$ & $110.0(6)$ & $\mathrm{O}(11)-\mathrm{Cl}(2)-\mathrm{O}(10)$ & 109.3(7) \\
\hline $\mathrm{O}(9)-\mathrm{Cl}(2)-\mathrm{O}(10)$ & $109.4(7)$ & $\mathrm{O}(12)-\mathrm{Cl}(2)-\mathrm{O}(10)$ & $110.4(6)$ \\
\hline
\end{tabular}

Symmetry transformations used to generate equivalent atoms: 
Table S3. Crystal data and structure refinement for 3, $\left[\operatorname{Hg}(\mathbf{1}) \mathrm{I}_{2}\right]_{2}$

Empirical formula

Formula weight

Temperature

Wavelength

Crystal system

Space group

Unit cell dimensions

Volume

Z

Density (calculated)

Absorption coefficient

$\mathrm{F}(000)$

Crystal size

Theta range for data collection

Index ranges

Reflections collected

Independent reflections

Completeness to theta $=27.00^{\circ}$

Absorption correction

Refinement method

Data / restraints / parameters

Goodness-of-fit on $\mathrm{F}^{2}$

Final $\mathrm{R}$ indices [I $>2 \operatorname{sigma}(\mathrm{I})]$

$\mathrm{R}$ indices (all data)

Largest diff. peak and hole
$\mathrm{C}_{64} \mathrm{H}_{64} \mathrm{Hg}_{2} \mathrm{I}_{4} \mathrm{~N}_{8} \mathrm{O}_{8} \mathrm{~S}_{4}$

2110.25

173(2) K

$0.71073 \AA$

Monoclinic

$\mathrm{P} 2(1) / \mathrm{c}$

$\mathrm{a}=11.3042(11) \AA \quad \alpha=90^{\circ}$.

$\mathrm{b}=36.375(4) \AA \quad \beta=100.603(2)^{\circ}$.

$\mathrm{c}=8.3764(8) \AA \quad \gamma=90^{\circ}$.

$3385.5(6) \AA^{3}$

2

$2.070 \mathrm{Mg} / \mathrm{m}^{3}$

$6.536 \mathrm{~mm}^{-1}$

2008

$0.40 \times 0.20 \times 0.15 \mathrm{~mm}^{3}$

1.12 to $27.00^{\circ}$.

$-14<=\mathrm{h}<=12,-46<=\mathrm{k}<=46,-10<=\mathrm{l}<=10$

20468

$7342[\mathrm{R}($ int $)=0.0626]$

$99.1 \%$

Empirical SADABS

Full-matrix least-squares on $\mathrm{F}^{2}$

7342 / 0 / 406

1.124

$\mathrm{R} 1=0.0435, \mathrm{wR} 2=0.1063$

$\mathrm{R} 1=0.0734, \mathrm{wR} 2=0.1283$

1.201 and -2.174 e. $\AA^{-3}$ 
Table S4. Bond lengths $(\AA)$ and angles $\left(^{\circ}\right)$ for $\mathbf{3},\left[\operatorname{Hg}(\mathbf{1}) \mathrm{I}_{2}\right]_{2}$

\begin{tabular}{|c|c|c|c|}
\hline $\mathrm{Hg}-\mathrm{S}(1)$ & $2.5983(18)$ & Hg-I(2) & $2.6920(6)$ \\
\hline $\mathrm{Hg}-\mathrm{I}(1) \# 1$ & $2.7080(6)$ & Hg-I(1) & $3.1541(7)$ \\
\hline $\mathrm{I}(1)-\mathrm{Hg} \# 1$ & $2.7080(6)$ & $S(1)-C(10)$ & $1.829(7)$ \\
\hline$S(1)-C(9)$ & $1.848(7)$ & $S(2)-C(25)$ & $1.825(8)$ \\
\hline$S(2)-C(26)$ & $1.827(7)$ & $\mathrm{O}(1)-\mathrm{C}(32)$ & $1.359(8)$ \\
\hline $\mathrm{O}(1)-\mathrm{C}(1)$ & $1.422(9)$ & $\mathrm{O}(2)-\mathrm{C}(3)$ & $1.354(8)$ \\
\hline $\mathrm{O}(2)-\mathrm{C}(2)$ & $1.440(8)$ & $\mathrm{O}(3)-\mathrm{N}(4)$ & $1.222(9)$ \\
\hline $\mathrm{O}(4)-\mathrm{N}(4)$ & $1.235(9)$ & $\mathrm{N}(1)-\mathrm{C}(12)$ & $1.383(10)$ \\
\hline $\mathrm{N}(1)-\mathrm{C}(24)$ & $1.454(10)$ & $\mathrm{N}(1)-\mathrm{C}(11)$ & $1.455(9)$ \\
\hline $\mathrm{N}(2)-\mathrm{N}(3)$ & $1.272(9)$ & $\mathrm{N}(2)-\mathrm{C}(15)$ & $1.410(9)$ \\
\hline $\mathrm{N}(3)-\mathrm{C}(18)$ & $1.427(10)$ & $\mathrm{N}(4)-\mathrm{C}(21)$ & $1.466(9)$ \\
\hline$C(1)-C(2)$ & $1.505(10)$ & $\mathrm{C}(3)-\mathrm{C}(4)$ & $1.407(10)$ \\
\hline$C(3)-C(8)$ & $1.432(10)$ & $C(4)-C(5)$ & $1.375(11)$ \\
\hline$C(5)-C(6)$ & $1.372(11)$ & $\mathrm{C}(6)-\mathrm{C}(7)$ & $1.404(11)$ \\
\hline$C(7)-C(8)$ & $1.369(10)$ & $\mathrm{C}(8)-\mathrm{C}(9)$ & $1.507(10)$ \\
\hline $\mathrm{C}(10)-\mathrm{C}(11)$ & $1.552(9)$ & $\mathrm{C}(12)-\mathrm{C}(13)$ & $1.406(11)$ \\
\hline$C(12)-C(17)$ & $1.410(10)$ & $C(13)-C(14)$ & $1.385(11)$ \\
\hline $\mathrm{C}(14)-\mathrm{C}(15)$ & $1.401(11)$ & $C(15)-C(16)$ & $1.396(11)$ \\
\hline$C(16)-C(17)$ & $1.384(10)$ & $\mathrm{C}(18)-\mathrm{C}(23)$ & $1.389(11)$ \\
\hline $\mathrm{C}(18)-\mathrm{C}(19)$ & $1.410(11)$ & $\mathrm{C}(19)-\mathrm{C}(20)$ & $1.369(11)$ \\
\hline$C(20)-C(21)$ & $1.382(11)$ & $\mathrm{C}(21)-\mathrm{C}(22)$ & $1.387(11)$ \\
\hline $\mathrm{C}(22)-\mathrm{C}(23)$ & $1.418(10)$ & $\mathrm{C}(24)-\mathrm{C}(25)$ & $1.529(11)$ \\
\hline$C(26)-C(27)$ & $1.495(10)$ & $\mathrm{C}(27)-\mathrm{C}(28)$ & $1.382(10)$ \\
\hline $\mathrm{C}(27)-\mathrm{C}(32)$ & $1.390(10)$ & $\mathrm{C}(28)-\mathrm{C}(29)$ & $1.395(11)$ \\
\hline $\mathrm{C}(29)-\mathrm{C}(30)$ & $1.378(11)$ & $\mathrm{C}(30)-\mathrm{C}(31)$ & $1.398(11)$ \\
\hline $\mathrm{C}(31)-\mathrm{C}(32)$ & $1.404(10)$ & & \\
\hline $\mathrm{S}(1)-\mathrm{Hg}-\mathrm{I}(2)$ & $108.90(4)$ & $\mathrm{S}(1)-\mathrm{Hg}-\mathrm{I}(1) \# 1$ & $118.01(4)$ \\
\hline $\mathrm{I}(2)-\mathrm{Hg}-\mathrm{I}(1) \# 1$ & $129.25(2)$ & $\mathrm{S}(1)-\mathrm{Hg}-\mathrm{I}(1)$ & $102.60(4)$ \\
\hline $\mathrm{I}(2)-\mathrm{Hg}-\mathrm{I}(1)$ & $97.72(2)$ & $\mathrm{I}(1) \# 1-\mathrm{Hg}-\mathrm{I}(1)$ & $90.291(18)$ \\
\hline $\mathrm{Hg} \# 1-\mathrm{I}(1)-\mathrm{Hg}$ & $89.709(18)$ & $\mathrm{C}(10)-\mathrm{S}(1)-\mathrm{C}(9)$ & $99.6(3)$ \\
\hline $\mathrm{C}(10)-\mathrm{S}(1)-\mathrm{Hg}$ & $102.1(2)$ & $\mathrm{C}(9)-\mathrm{S}(1)-\mathrm{Hg}$ & $107.5(3)$ \\
\hline$C(25)-S(2)-C(26)$ & $102.7(4)$ & $\mathrm{C}(32)-\mathrm{O}(1)-\mathrm{C}(1)$ & $119.0(6)$ \\
\hline $\mathrm{C}(3)-\mathrm{O}(2)-\mathrm{C}(2)$ & $118.1(6)$ & $\mathrm{C}(12)-\mathrm{N}(1)-\mathrm{C}(24)$ & $120.8(6)$ \\
\hline $\mathrm{C}(12)-\mathrm{N}(1)-\mathrm{C}(11)$ & $121.6(6)$ & $\mathrm{C}(24)-\mathrm{N}(1)-\mathrm{C}(11)$ & $117.4(6)$ \\
\hline $\mathrm{N}(3)-\mathrm{N}(2)-\mathrm{C}(15)$ & $112.9(6)$ & $\mathrm{N}(2)-\mathrm{N}(3)-\mathrm{C}(18)$ & $113.5(6)$ \\
\hline $\mathrm{O}(3)-\mathrm{N}(4)-\mathrm{O}(4)$ & $123.4(7)$ & $\mathrm{O}(3)-\mathrm{N}(4)-\mathrm{C}(21)$ & $118.3(7)$ \\
\hline $\mathrm{O}(4)-\mathrm{N}(4)-\mathrm{C}(21)$ & $118.3(7)$ & $\mathrm{O}(1)-\mathrm{C}(1)-\mathrm{C}(2)$ & $107.2(6)$ \\
\hline $\mathrm{O}(2)-\mathrm{C}(2)-\mathrm{C}(1)$ & $106.3(6)$ & $\mathrm{O}(2)-\mathrm{C}(3)-\mathrm{C}(4)$ & $125.9(6)$ \\
\hline \multirow[t]{2}{*}{$\mathrm{O}(2)-\mathrm{C}(3)-\mathrm{C}(8)$} & $114.8(6)$ & $\mathrm{C}(4)-\mathrm{C}(3)-\mathrm{C}(8)$ & $119.3(6)$ \\
\hline & & S11 & \\
\hline
\end{tabular}




\begin{tabular}{llll}
$\mathrm{C}(5)-\mathrm{C}(4)-\mathrm{C}(3)$ & $119.0(7)$ & $\mathrm{C}(6)-\mathrm{C}(5)-\mathrm{C}(4)$ & $122.2(8)$ \\
$\mathrm{C}(5)-\mathrm{C}(6)-\mathrm{C}(7)$ & $119.5(7)$ & $\mathrm{C}(8)-\mathrm{C}(7)-\mathrm{C}(6)$ & $120.5(7)$ \\
$\mathrm{C}(7)-\mathrm{C}(8)-\mathrm{C}(3)$ & $119.5(7)$ & $\mathrm{C}(7)-\mathrm{C}(8)-\mathrm{C}(9)$ & $122.3(7)$ \\
$\mathrm{C}(3)-\mathrm{C}(8)-\mathrm{C}(9)$ & $118.1(6)$ & $\mathrm{C}(8)-\mathrm{C}(9)-\mathrm{S}(1)$ & $110.8(5)$ \\
$\mathrm{C}(11)-\mathrm{C}(10)-\mathrm{S}(1)$ & $110.4(5)$ & $\mathrm{N}(1)-\mathrm{C}(11)-\mathrm{C}(10)$ & $112.0(6)$ \\
$\mathrm{N}(1)-\mathrm{C}(12)-\mathrm{C}(13)$ & $121.8(7)$ & $\mathrm{N}(1)-\mathrm{C}(12)-\mathrm{C}(17)$ & $121.8(7)$ \\
$\mathrm{C}(13)-\mathrm{C}(12)-\mathrm{C}(17)$ & $116.4(7)$ & $\mathrm{C}(14)-\mathrm{C}(13)-\mathrm{C}(12)$ & $122.0(7)$ \\
$\mathrm{C}(13)-\mathrm{C}(14)-\mathrm{C}(15)$ & $120.7(7)$ & $\mathrm{C}(16)-\mathrm{C}(15)-\mathrm{C}(14)$ & $118.1(7)$ \\
$\mathrm{C}(16)-\mathrm{C}(15)-\mathrm{N}(2)$ & $117.5(7)$ & $\mathrm{C}(14)-\mathrm{C}(15)-\mathrm{N}(2)$ & $124.4(7)$ \\
$\mathrm{C}(17)-\mathrm{C}(16)-\mathrm{C}(15)$ & $121.0(7)$ & $\mathrm{C}(16)-\mathrm{C}(17)-\mathrm{C}(12)$ & $121.8(7)$ \\
$\mathrm{C}(23)-\mathrm{C}(18)-\mathrm{C}(19)$ & $119.6(7)$ & $\mathrm{C}(23)-\mathrm{C}(18)-\mathrm{N}(3)$ & $125.1(7)$ \\
$\mathrm{C}(19)-\mathrm{C}(18)-\mathrm{N}(3)$ & $115.2(7)$ & $\mathrm{C}(20)-\mathrm{C}(19)-\mathrm{C}(18)$ & $121.1(8)$ \\
$\mathrm{C}(19)-\mathrm{C}(20)-\mathrm{C}(21)$ & $119.0(7)$ & $\mathrm{C}(20)-\mathrm{C}(21)-\mathrm{C}(22)$ & $122.1(7)$ \\
$\mathrm{C}(20)-\mathrm{C}(21)-\mathrm{N}(4)$ & $119.5(7)$ & $\mathrm{C}(22)-\mathrm{C}(21)-\mathrm{N}(4)$ & $118.4(7)$ \\
$\mathrm{C}(21)-\mathrm{C}(22)-\mathrm{C}(23)$ & $118.7(7)$ & $\mathrm{C}(18)-\mathrm{C}(23)-\mathrm{C}(22)$ & $119.4(7)$ \\
$\mathrm{N}(1)-\mathrm{C}(24)-\mathrm{C}(25)$ & $114.0(6)$ & $\mathrm{C}(24)-\mathrm{C}(25)-\mathrm{S}(2)$ & $111.1(6)$ \\
$\mathrm{C}(27)-\mathrm{C}(26)-\mathrm{S}(2)$ & $112.4(5)$ & $\mathrm{C}(28)-\mathrm{C}(27)-\mathrm{C}(32)$ & $119.3(7)$ \\
$\mathrm{C}(28)-\mathrm{C}(27)-\mathrm{C}(26)$ & $120.5(7)$ & $\mathrm{C}(32)-\mathrm{C}(27)-\mathrm{C}(26)$ & $120.2(6)$ \\
$\mathrm{C}(27)-\mathrm{C}(28)-\mathrm{C}(29)$ & $121.6(7)$ & $\mathrm{C}(30)-\mathrm{C}(29)-\mathrm{C}(28)$ & $118.6(7)$ \\
$\mathrm{C}(29)-\mathrm{C}(30)-\mathrm{C}(31)$ & $121.4(7)$ & $\mathrm{C}(30)-\mathrm{C}(31)-\mathrm{C}(32)$ & $118.9(7)$ \\
$\mathrm{O}(1)-\mathrm{C}(32)-\mathrm{C}(27)$ & $115.3(6)$ & $\mathrm{O}(1)-\mathrm{C}(32)-\mathrm{C}(31)$ & $124.5(7)$ \\
$\mathrm{C}(27)-\mathrm{C}(32)-\mathrm{C}(31)$ & $120.3(7)$ & & \\
\hline
\end{tabular}

Symmetry transformations used to generate equivalent atoms:

$\# 1-\mathrm{x},-\mathrm{y}+1,-\mathrm{z}+2$ 


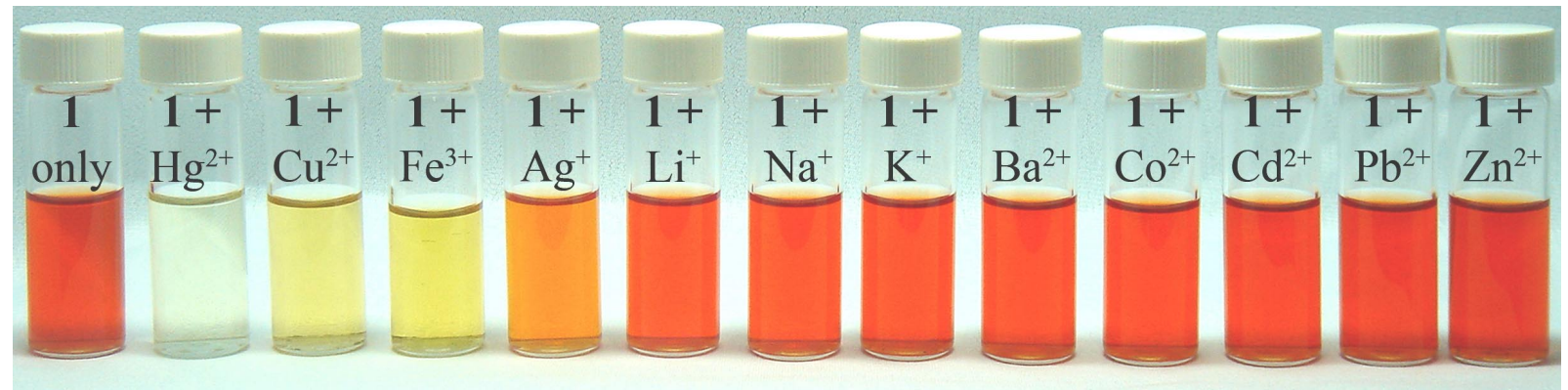

Figure S1. Metal-induced color changes of 1 in acetonitrile (5 equiv of metal nitrate was added). 


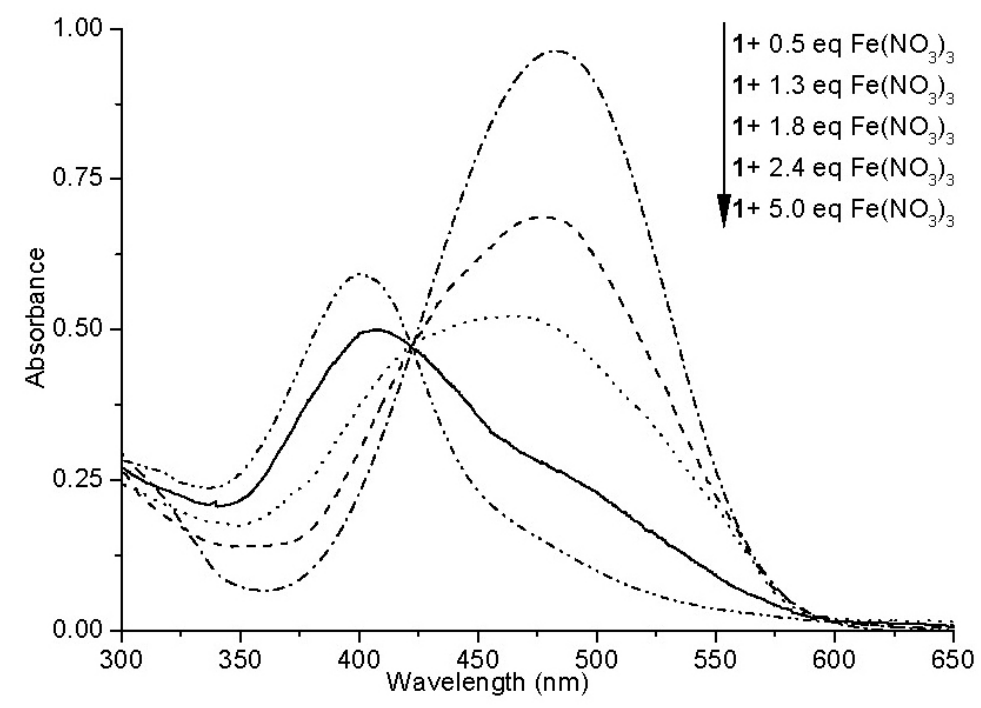

Figure S2. UV/Vis titrations of $\mathbf{1}(0.30 \mathrm{mM})$ with $\mathrm{Fe}\left(\mathrm{NO}_{3}\right)_{3}(0-5.0$ equiv) in acetonitrile. 


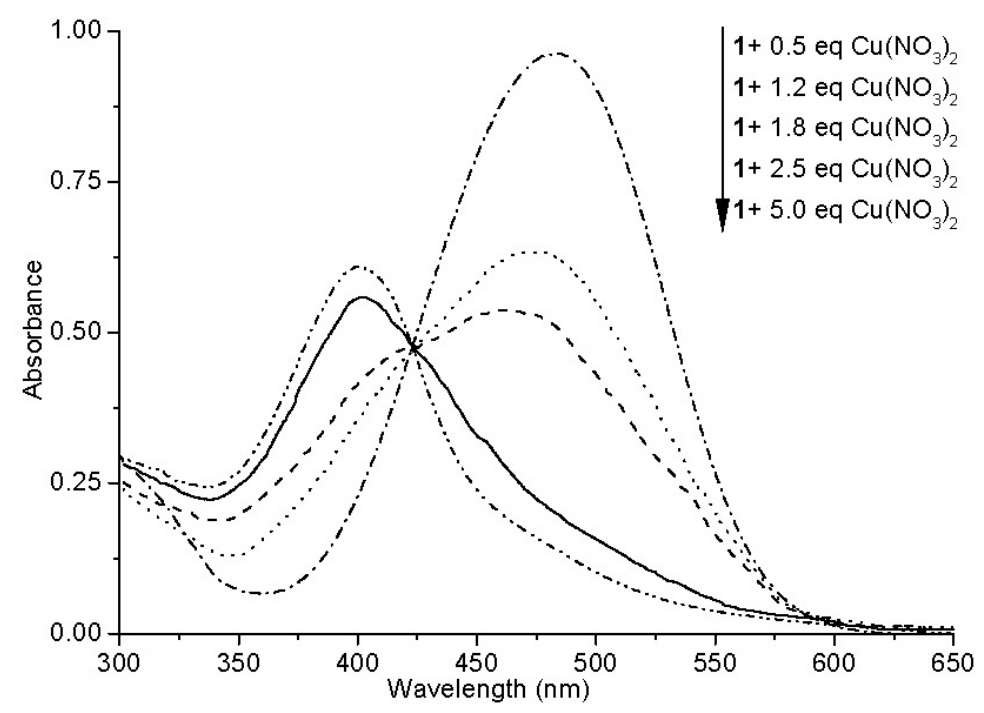

Figure S3. UV/Vis titrations of $1(0.30 \mathrm{mM})$ with $\mathrm{Cu}\left(\mathrm{NO}_{3}\right)_{2}(0-5.0$ equiv) in acetonitrile. 


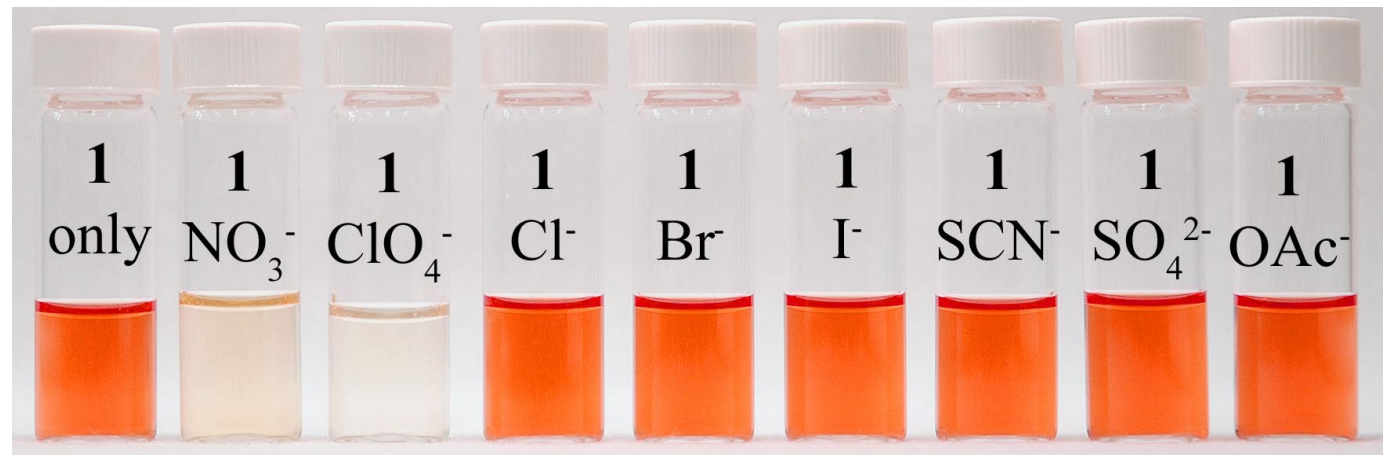

Figure S4. Anion-controlled color changes of 1in acetonitrile (5 equiv of mercury(II) salt was added). 


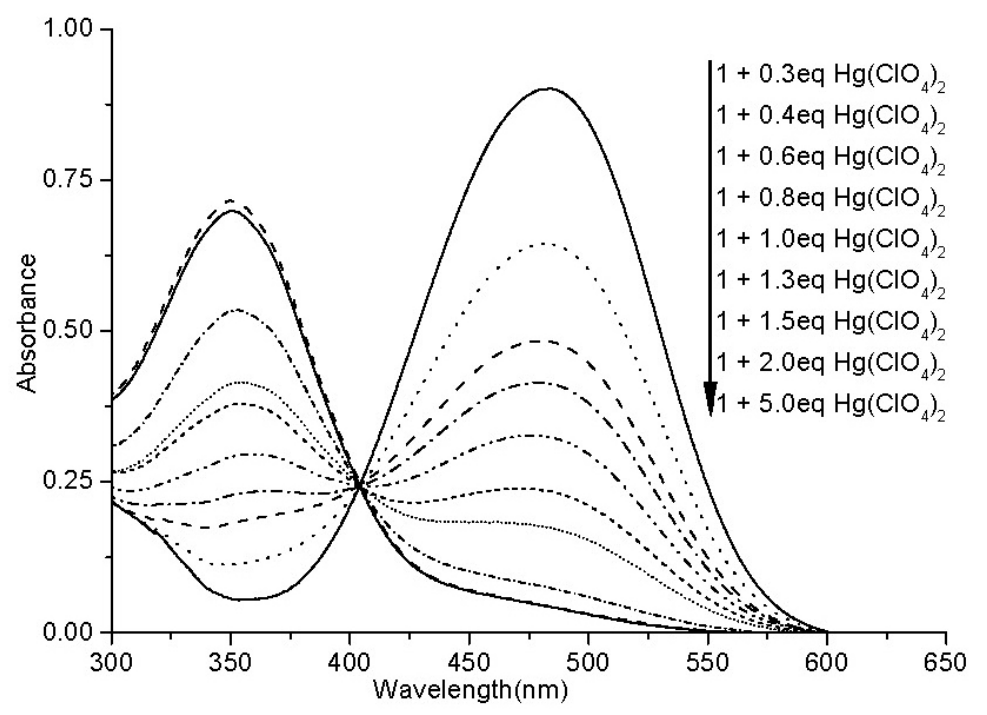

Figure S5. UV/Vis titrations of $1(0.30 \mathrm{mM})$ with $\mathrm{Hg}\left(\mathrm{ClO}_{4}\right)_{2}(0-5.0$ equiv) in acetonitrile. 
Figure S6. ${ }^{1} \mathrm{H}$ NMR spectrum of $\mathbf{1}$

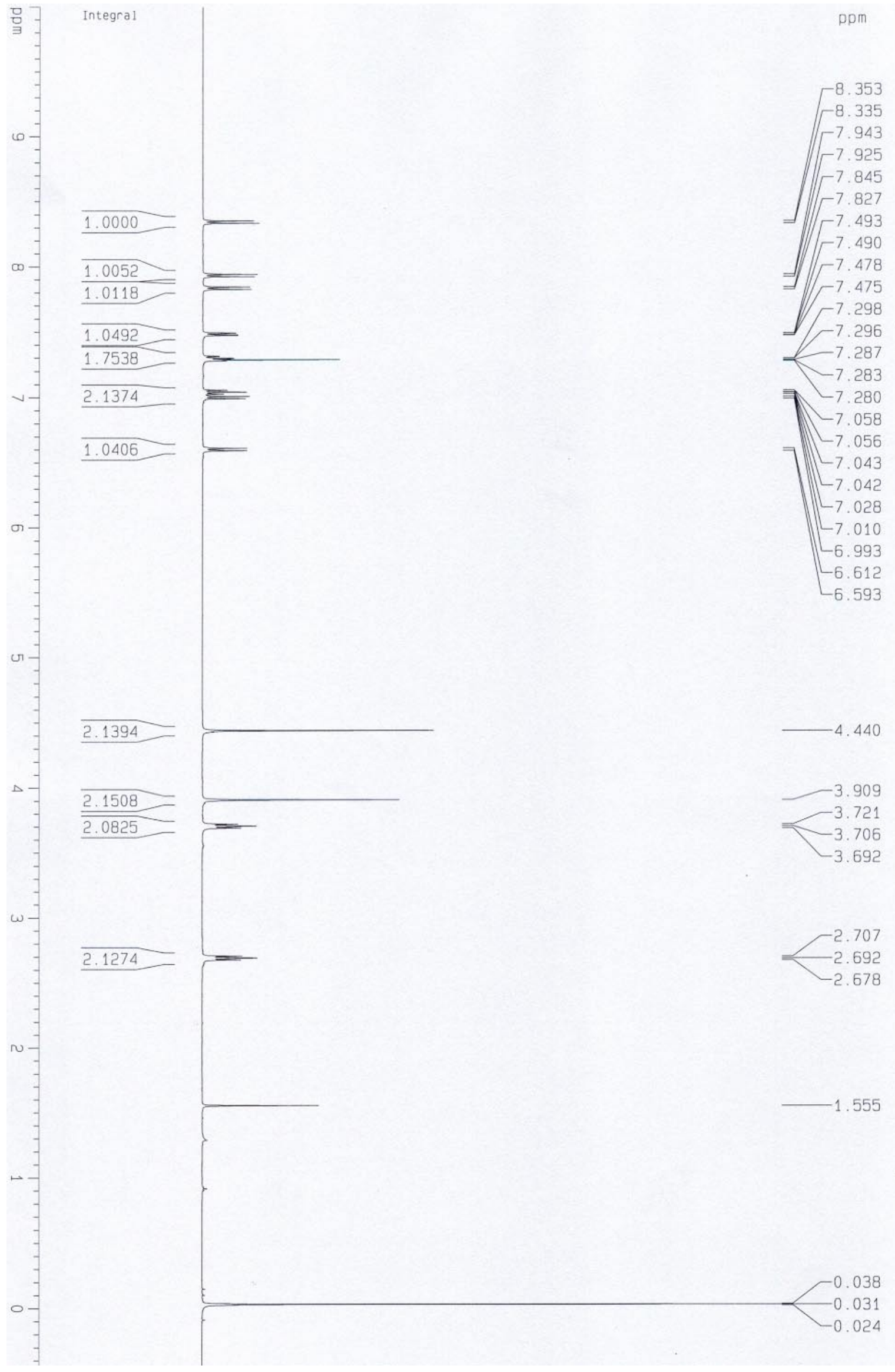


Figure S7. ${ }^{13} \mathrm{C}$ NMR spectrum of $\mathbf{1}$

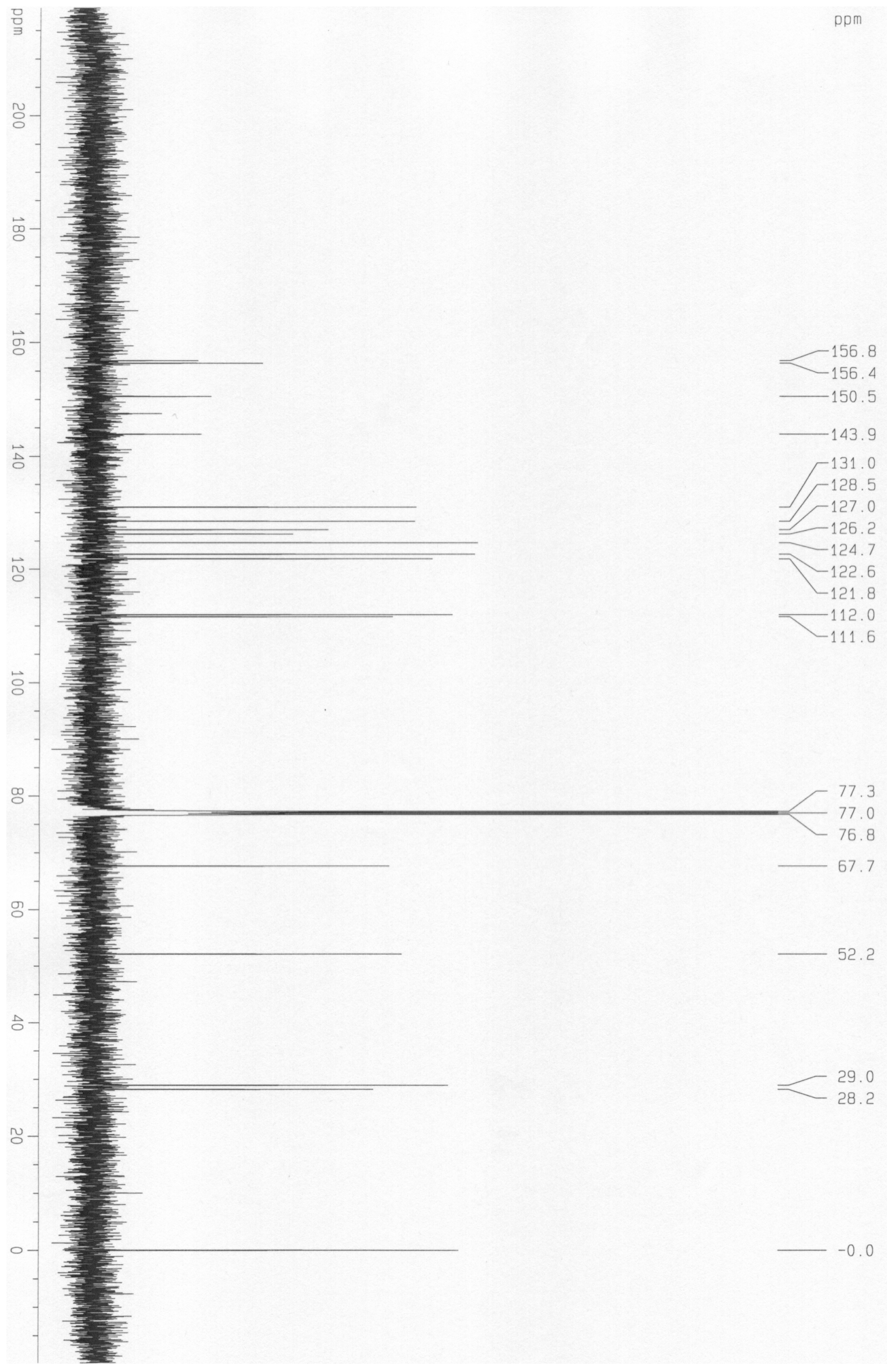


Figure S8. ${ }^{1} \mathrm{H}$ NMR spectrum of 4

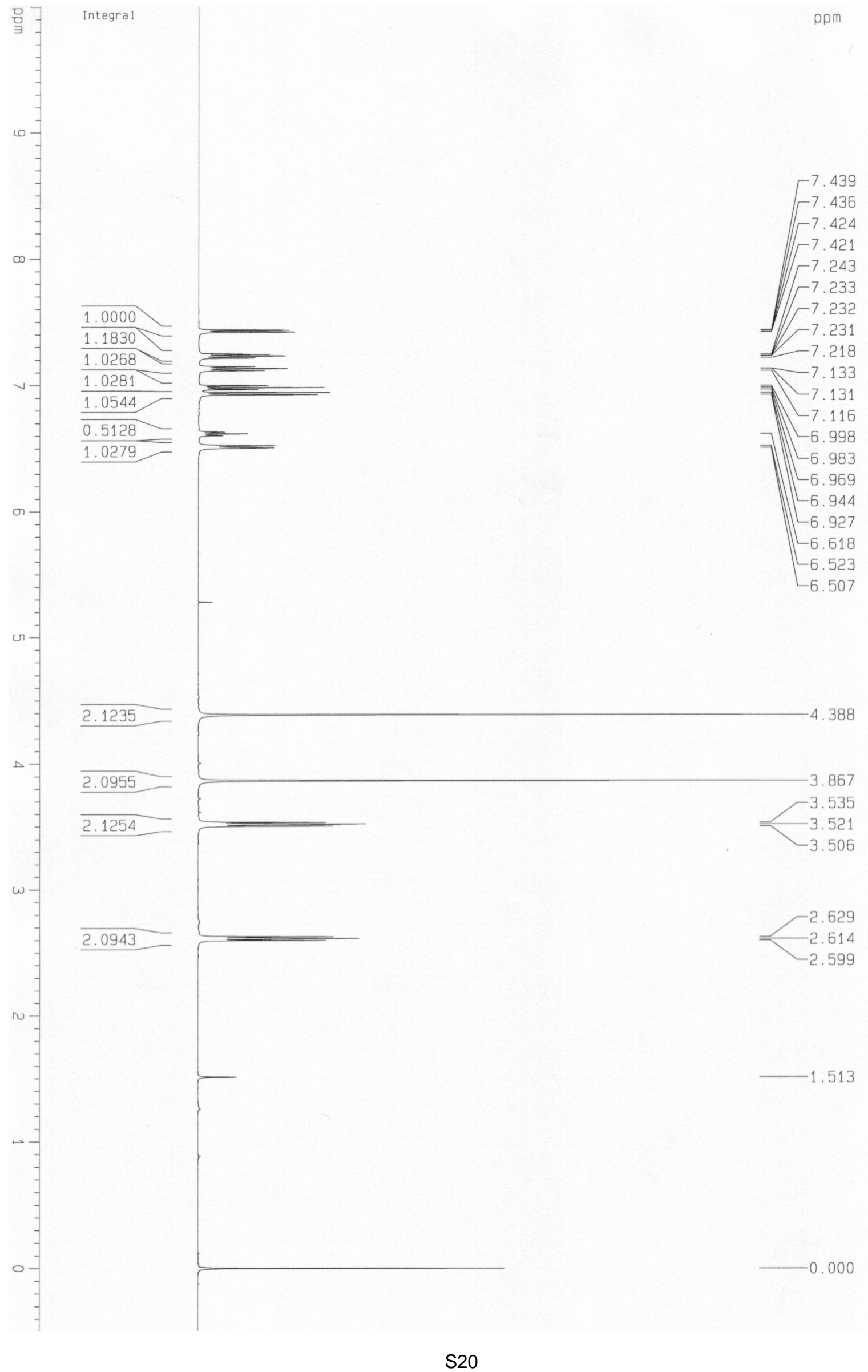


Figure S9. ${ }^{13} \mathrm{C}$ NMR spectrum of 4

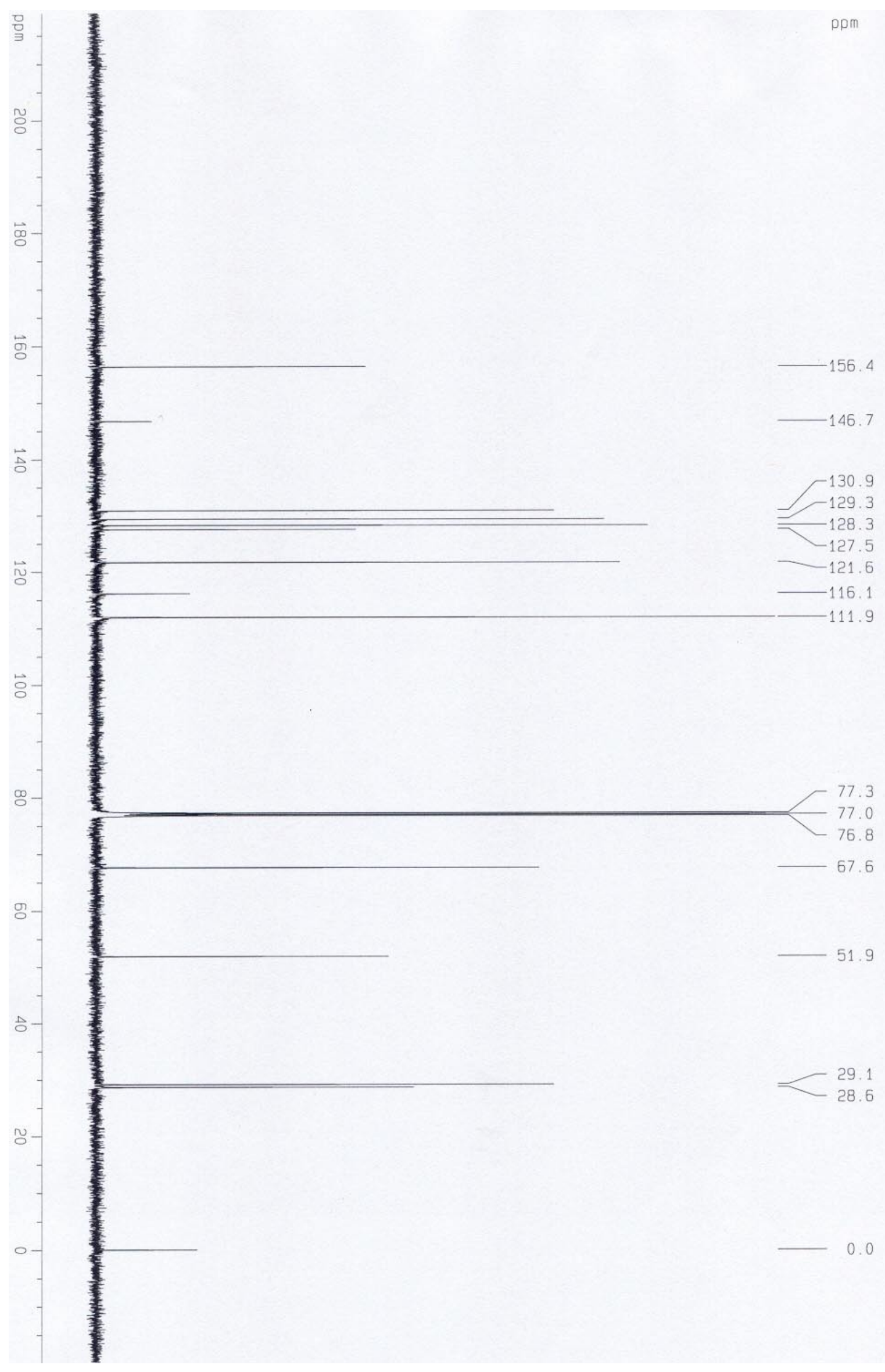

